\title{
Epidural analgesia during labour and autism risk: getting lost on the causal path
}

\author{
François Martin Carrier, MD, MSc, FRCPC (1) Anne Lavoie, MD, FRCPC • Valerie Zaphiratos, MD, MSc, \\ FRCPC
}

Received: 11 November 2020/Revised: 13 November 2020/Accepted: 13 November 2020/Published online: 6 January 2021

(C) Canadian Anesthesiologists' Society 2021

In Canada, over $60 \%$ of labouring women choose labour epidural analgesia (LEA). ${ }^{1}$ Innovation in obstetric anesthesia care, including the use of LEA, has permitted an increasing number of parturients with major comorbidities to safely go through labour and delivery. Although much of LEA research has focused on perinatal and obstetric outcomes, the long-term effects of LEA on mothers and infants need further research. ${ }^{2}$ The increased incidence of autism over the past years has motivated research efforts to find cause. ${ }^{3}$ Despite these efforts, its underlying etiology remains poorly understood.

\footnotetext{
F. M. Carrier, MD, MSc, FRCPC ( $\square)$

Department of Anesthesiology and Department of Medicine Critical Care Division, Centre hospitalier de l'Université de Montréal (CHUM), 900, rue Saint-Denis, porte S03-434, Montréal, QC H2X 0A9, Canada

e-mail: francois.martin.carrier@umontreal.ca
}

Centre de Recherche du Centre hospitalier de l'Université de Montréal, Montréal, QC, Canada

\author{
A. Lavoie, MD, FRCPC \\ Department of Anesthesiology, Centre hospitalier de \\ l'Université de Montréal, Montréal, QC, Canada
}

\section{Zaphiratos, MD, MSc, FRCPC}

Department of Anesthesiology, Hôpital Maisonneuve-Rosemont, Montréal, QC, Canada

Obstetric Section, Canadian Anesthesiologists' Society, Toronto, ON, Canada

\section{Labour epidural analgesia and autism - a confounded association}

In a recent article in JAMA Pediatrics, Qiu et al. reported an association between LEA and autism in the offspring, after adjustment for potential confounders. ${ }^{4}$ Although we congratulate these authors for their efforts in trying to delineate an association between a perinatal variable (i.e., LEA) and a long-term pediatric outcome (i.e., autism), we believe that their reported association cannot be interpreted as causal and has limited clinical applicability. As mentioned in a subsequent joint position statement from the Canadian Anesthesiologists' Society (CAS), the Society of Obstetricians and Gynaecologists of Canada, and the Canadian Paediatric Society, randomizedcontrolled experimental designs (and their meta-analyses) provide the strongest evidence for causal relationships. ${ }^{5}$ Nevertheless, in obstetric anesthesia, such trials comparing LEA with no LEA would neither be feasible nor ethical. Drawing causal inference from observational data to explore research questions, although not always ideal, is thus required. ${ }^{6}$

Different epidemiological models and statistical techniques have been proposed over the years to better identify causal relationships from observational data. ${ }^{7}$ The Inverse Probability of Treatment Weighting analysis, as used in the Qiu et al. study, is one such technique that focuses results on one estimated association and minimizes model assumptions. Nevertheless, its interpretation is based on an important assumption-i.e., all confounders of the association of interest are known, adequately measured, and included in the model. In their study, the authors included many important confounders, but others were not sufficiently addressed or considered. Residual and 
uncontrolled confounding provides biased estimates that cannot suggest causation.

The authors reported the E-value, a recently described quantification of the minimum strength of association on the risk ratio scale that an uncontrolled confounder must have with both the exposure and the outcome to fully explain an observed association. ${ }^{8}$ They suggested that any uncontrolled confounder would have to be associated with both LEA and autism with a relative risk of 1.76 to explain the observed association, which in their view is unlikely. Nevertheless, in our opinion, significant confounders likely existed in either the baseline health determinants or aspects of labour itself, and the reality is likely far different than a simple biased association between LEA and autism.

\section{The LEA "choice" - a confusing assumption}

Labour epidural analgesia is a dynamic shared-decision process between the patient and the provider and depends on many bio-psychosocial phenomena, including pain, comorbidities, and personal preference. These variables in turn are influenced by socioeconomic status, education, religious beliefs, and overall lifestyle habits-all variables that might explain observed pediatric outcomes. For example, severe pain due to labour dystocia is associated with both an increased risk for LEA and poorer neonatal outcomes. Psychological and cultural interpretations of pain are also known to contribute to disparities regarding labour analgesia and may influence other health determinants. ${ }^{9}$ These factors create a network of complex interrelated variables that renders causality between LEA and neonatal outcomes difficult to understand (Figure). The authors did not adjust for such confounders or many other obstetric factors, which could explain the observed association between the use of LEA and autism.

\section{Confounders and outcome classification bias}

In this issue of the Journal, Glezerson et al. also reported similar concerns regarding uncontrolled confounders, particularly those associated with the indication of LEA. ${ }^{10}$ The authors rightfully argued that some variables remained unbalanced across groups, in line with the epidemiological definition of uncontrolled confounding we discussed above. Specifically, they proposed that outcome classification bias occurred from using a large database with limited predictive values in the context of rare events-a bias that precludes interpretability of the results. Nevertheless, their proposed explanation of nondifferential outcome misclassification would provide biased results toward the null value (i.e., no difference between groups). To bias results away from the null value (i.e., away from a relative risk of 1), misclassification has to be differential (i.e., misclassify the outcome preferentially in one exposure group). Since it is highly unlikely that the use of LEA itself may have affected the diagnosis of autism, the proposed effect of misclassification bias on the observed spurious association may still stand if a health determinant associated with the use of LEA influenced the autism diagnosis process and thus outcome classification. To a certain extent, such bias rebounds the problem back to uncontrolled confounding and causation assumptions.

\section{What is causation?}

Many scientists have tried over the years to circumscribe causality within different frameworks. Sir Austin Bradford Hill published criteria that are well-known and were used by the US Surgeon General in the 1960's to first establish causality between tobacco exposure and lung cancer. ${ }^{11}$ These criteria propose a causality assessment through a qualitative analysis of observed associations that may be viewed as circumventing the problem of uncontrolled confounding. Some of these criteria were either addressed, explored, or mentioned in the recently published paper, but many others were not (Table). ${ }^{4}$ There is a temporal relationship between LEA and autism, since one occurs before the other. The authors found a significant doseresponse relationship, which is an important causal criterion. The authors exposed the biological plausibility of this association by reporting translational data on local anesthetic toxicity and the developing brain. Nevertheless, these data are sparse, mostly involve animal studies, and use local anesthetic doses that are many times greater than what is used in clinical practice. Also, their observed association is weak, has not been observed elsewhere, is not supported by any experimental data, and notably, as previously discussed, is biased by alternative causal pathways, including their dose-response exploratory analyses that were not deconfounded, akin to their main analyses. As mentioned, more recent work addressed causation through a more quantitative lens, emphasizing the fact that confounders are non-random variables associated with both exposure and outcomes that may explain many reported observations. ${ }^{6}$ In modern causal inference, such confounders must be fully addressed, notwithstanding criteria proposed by Bradford Hill, before establishing causation. 


\section{Health impacts of spurious correlations}

Non-causal epidemiological and ecological associations are common. Vaccines and autism is a well-known example that created 20 years of debate and still has an impact on public health interventions. ${ }^{12}$ In obstetrics, the association between parity and the incidence of Down syndrome in the offspring has also spurred debate. ${ }^{13}$ The unbiased reality of this association is not due to complex physiologic disturbances that occur during subsequent pregnancies, rather it is explained by maternal age, which increases with parity. ${ }^{13}$ It was once believed that LEA was associated with an increased rate of Cesarean delivery, but this is now recognized as a non-causal association. ${ }^{14}$ Like the aforementioned associations, many factors were at play in this association. For example, dystocic labour increases the risk of Cesarean delivery, and is also associated with increased labour pain, thus increasing the likelihood of requesting LEA. Caution must thus be advised when conducting analyses from observational data. Adjusting for confounders is challenging, yet crucial to draw meaningful conclusions from retrospective studies. Otherwise, spurious correlations, thought to be causal, may lead to inappropriate health interventions, patient anxiety, or even costly interventions that do not benefit patients or healthcare providers.

\section{The anesthesia community reacts}

On the same day the Qiu et al. study was published, a joint statement was released by the Society for Obstetric Anesthesia and Perinatology, the American Society of Anesthesiologists, the Society for Pediatric Anesthesia, the American College of Obstetricians and Gynecologists and the Society for Maternal-Fetal Medicine to reassure women that the association between LEA and autism does not imply causation. ${ }^{4,15}$ The same week, the Royal College of Anesthetists and the CAS also emitted statements to assure women that this study lacked causation and, importantly, did not change current clinical practice recommendations regarding epidural analgesia for labour pain. ${ }^{5,16}$ These statements reflect leading experts' opinion, established from vast academic and clinical experience, that the reported association is flawed, not clinically meaningful and potentially harmful for women.

Analyzing and reporting the effects of LEA on longterm outcomes is needed. The pathophysiology of autism is not fully understood and exploring epidemiological associations to identify potential causes are also needed. Exposure to LEA involves a multifaceted decision-making process that renders the analysis of its role in autism hard to circumscribe. Publishing specious associations between
LEA and obstetrical outcomes may impact women and their choice of pain management, and their willingness to adhere to a complex obstetrical plan that frequently includes LEA. Such choices may lead to potential harm and unnecessary guilt, especially in vulnerable subgroups of the population. To prevent the dissemination of such spurious correlations, association analyses should not be conducted outside of a clear causal framework that includes all important and well-measured confounders. Otherwise, scientists may easily lose themselves on the causal path.

\section{Analgésie péridurale pendant le travail obstétrical et risque d'autisme, ou comment se perdre sur le chemin de la causalité}

Au Canada, plus de $60 \%$ des femmes en travail choisissent une analgésie péridurale obstétricale (APO). ${ }^{1}$ L'innovation dans les soins obstétricaux d'anesthésie, notamment l'utilisation de l'APO, a permis à un nombre croissant de parturientes présentant d'importantes comorbidités d'avoir un travail et un accouchement en toute sécurité. Bien qu'une grande partie de la recherche sur l'APO se soit concentrée sur les devenirs périnataux et obstétricaux, les effets à long terme de la l'APO sur les mères et les nourrissons doivent faire l'objet d'autres recherches. ${ }^{2}$ L'incidence accrue de l'autisme au cours des dernières années a motivé les efforts de recherche pour en trouver la cause. ${ }^{3}$ Malgré ces efforts, l'étiologie sous-jacente de l'autisme demeure mal comprise.

\section{Analgésie péridurale obstétricale et autisme - une association confuse}

Dans un article récent paru dans JAMA Pediatrics, après ajustement pour tenir compte des facteurs confondants potentiels, Qiu et coll. ont rapporté une association entre l'APO et l'autisme chez les enfants. ${ }^{4}$ Bien que nous félicitions ces auteurs pour leurs efforts pour essayer de décrire une association entre une variable périnatale (c.-àd. l'APO) et un devenir pédiatrique à long terme (c.-à-d., l'autisme), nous pensons que l'association rapportée ne peut être interprétée comme étant causale et qu'elle n'a qu'une applicabilité clinique limitée. Comme déjà mentionné dans une déclaration commune de position subséquente de la Société canadienne des anesthésiologistes (SCA), de la Société des obstétriciens et gynécologues du Canada et de la Société canadienne de 
Table Causality according to the main criteria reported by Sir Bradford Hill

\begin{tabular}{|c|c|}
\hline Criteria & Results \\
\hline Experimental data & None exists \\
\hline Temporal sequence & LEA occurred before autism \\
\hline $\begin{array}{l}\text { Strength of } \\
\text { association }\end{array}$ & Weak association \\
\hline $\begin{array}{l}\text { Dose-response } \\
\text { effect }\end{array}$ & $\begin{array}{l}\text { The longer the exposure to LEA, the higher the } \\
\text { risk }\end{array}$ \\
\hline $\begin{array}{l}\text { Biological } \\
\text { plausibility }\end{array}$ & Few translational data \\
\hline Consistency & First report of this association \\
\hline
\end{tabular}

Coherence, analogy, and specificity are other reported criteria that are often considered not more informative than the main six ones. LEA = labour epidural analgesia.

pédiatrie, les études randomisées contrôlées de nature expérimentale (et leurs méta-analyses) fournissent les données probantes les plus rigoureuses en ce qui a trait aux relations causales. ${ }^{5}$ Néanmoins, en anesthésie obstétricale, de tels études comparant une APO à son absence ne seraient ni réalisables ni éthiques. Il est donc nécessaire de recourir à une inférence causale à partir de données observationnelles pour explorer les questions de recherche, bien que cette méthodologie ne soit pas toujours idéale. $^{6}$

Au fil des ans, différents modèles épidémiologiques et techniques statistiques ont été proposés afin de mieux identifier les relations causales à partir de données observationnelles. $^{7}$ L'analyse de la pondération par l'inverse de la probabilité de traitement, telle qu'utilisée dans l'étude de Qiu et coll., est l'une de ces techniques qui base les résultats sur une association estimée et minimise les hypothèses du modèle. Néanmoins, son interprétation est fondée sur une hypothèse importante, soit que tous les facteurs confondants de l'association de l'étude sont connus, mesurés adéquatement et inclus dans le modèle. Dans leur étude, les auteurs ont inclus de nombreux facteurs confondants importants, alors que d'autres n'ont pas été suffisamment abordés ou pris en considération. Les facteurs de confusion résiduels et non contrôlés fournissent des estimations biaisées qui ne peuvent suggérer de causalité.

A A simple biased causal path.

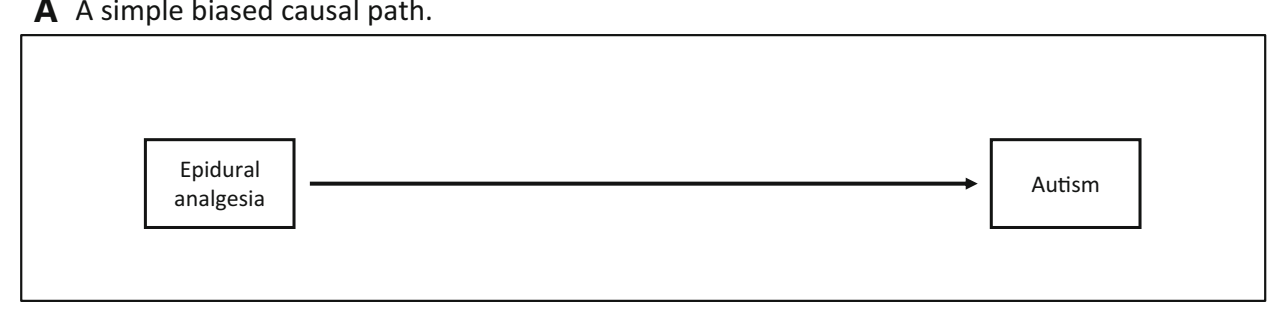

B A more realistic but yet incomplete complex causal framework.

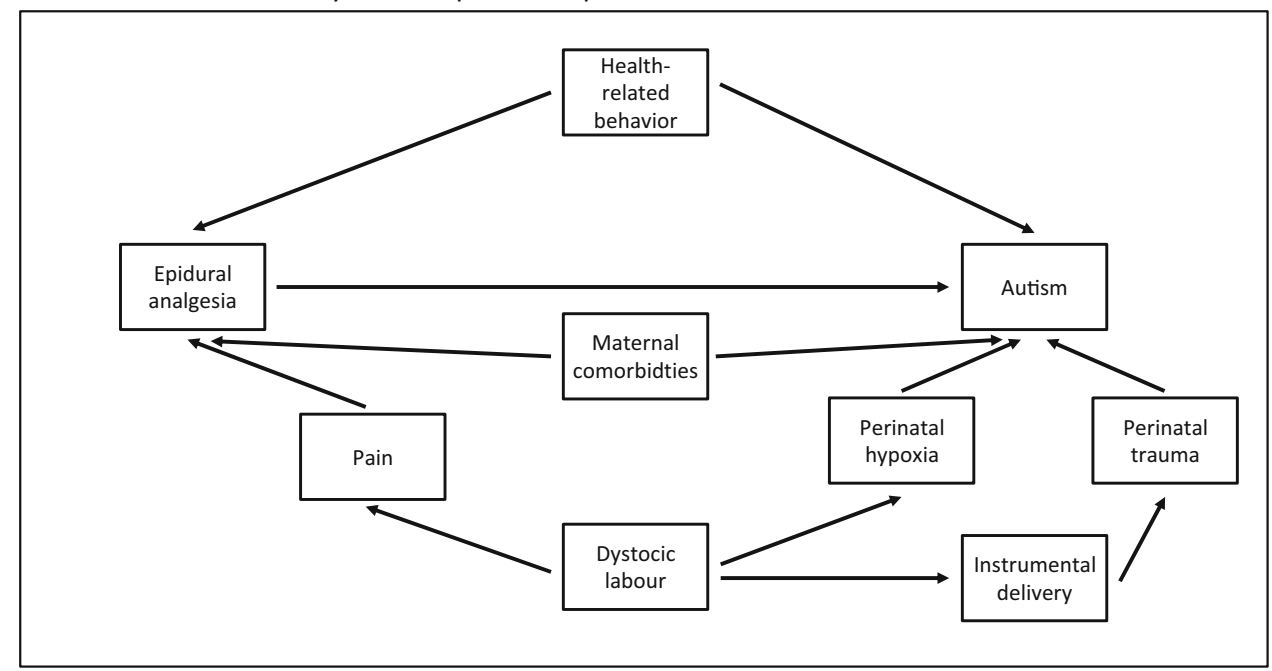

Arrows indicate the link from a cause to its effect.

Figure Causal path between epidural analgesia and autism 
Les auteurs ont rapporté la valeur $\mathrm{E}$, une quantification récemment décrite de la force minimale d'association sur l'échelle du risque relatif qu'un facteur confondant non contrôlé doit avoir à la fois avec l'exposition et avec le résultat pour expliquer pleinement une association observée. ${ }^{8}$ Ils ont suggéré que tout facteur confondant non contrôlé devrait être associé à la fois à l'APO et à l'autisme avec un risque relatif de 1,76 pour expliquer l'association observée, ce qui, à leur avis, serait peu probable. Néanmoins, à notre avis, d'importants facteurs confondants existaient probablement, que ce soit dans les déterminants de santé de base ou dans certains aspects du travail obstétrical lui-même, et la réalité est probablement bien différente d'une simple association biaisée entre l'APO et l'autisme.

\section{Le « choix » de l'APO — une présomption confondante}

L'analgésie péridurale obstétricale est un processus dynamique de prise de décision partagée entre la patiente et le fournisseur de soins qui dépend de nombreux phénomènes bio-psycho-sociaux, notamment la douleur, les comorbidités et les préférences personnelles. Ces variables sont à leur tour influencées par le statut socioéconomique, l'éducation, les croyances religieuses et les habitudes de vie globales - autant de variables qui pourraient expliquer les devenirs pédiatriques observés. Par exemple, la douleur intense causée par une dystocie du travail est associée à la fois à un risque accru d'APO et à des devenirs néonataux moins bons. Il a également été observé que les interprétations psychologiques et culturelles de la douleur contribuaient aux disparités en ce qui concerne l'analgésie pour le travail et pouvaient influencer d'autres déterminants de santé. ${ }^{9}$ Ces facteurs créent un réseau de variables interdépendantes complexes qui rend difficile à comprendre la causalité entre l'APO et les devenirs néonataux (voir figure). Les auteurs n'ont pas ajusté leurs résultats pour tenir compte de tels facteurs confondants ou de bien d'autres facteurs obstétricaux, ce qui pourrait expliquer l'association observée entre le recours à une APO et l'autisme.

\section{Facteurs confondants et biais de classification des devenirs}

Dans ce numéro du Journal, Glezerson et coll. ont également fait état de préoccupations similaires concernant des facteurs confondants non contrôlés, en particulier ceux associées à une indication d'APO.$^{10}$ Les auteurs ont soutenu, à juste titre, que certaines variables demeuraient déséquilibrées entre les groupes, ce qui est
Tableau Liens de causalité selon les principaux critères rapportés par Sir Bradford Hill

\begin{tabular}{|c|c|}
\hline Critères & Résultats \\
\hline $\begin{array}{l}\text { Données } \\
\text { expérimentales }\end{array}$ & Aucune n'existe \\
\hline $\begin{array}{l}\text { Séquence } \\
\text { temporelle }\end{array}$ & L'APO est survenue avant l'autisme \\
\hline $\begin{array}{l}\text { Force de } \\
\text { l'association }\end{array}$ & Association faible \\
\hline $\begin{array}{l}\text { Effet dose- } \\
\text { réponse }\end{array}$ & $\begin{array}{l}\text { Plus l'exposition à l'APO est prolongée, plus le } \\
\text { risque est élevé }\end{array}$ \\
\hline $\begin{array}{l}\text { Plausibilité } \\
\text { biologique }\end{array}$ & Peu de données translationnelles \\
\hline Consistance & Première mention de cette association \\
\hline
\end{tabular}

La cohérence, l'analogie et la spécificité sont d'autres critères rapportés souvent considérés comme moins instructifs que les six principaux. $\mathrm{APO}=$ analgésie péridurale obstétricale.

conforme à la définition épidémiologique de facteur confondant non contrôlé discutée ci-dessus. Plus précisément, ils ont proposé que le biais de classification des devenirs soit survenu à cause de l'utilisation d'une vaste base de données avec des valeurs prédictives limitées dans le contexte d'événements rares - un biais qui exclut l'interprétation des résultats. Néanmoins, l'explication proposée d'une classification erronée non différentielle des devenirs donnerait des résultats biaisés en faveur d'une valeur nulle (c.-à-d. aucune différence entre les groupes). Pour écarter les résultats d'une valeur nulle (c.-à-d. loin d'un risque relatif de 1), la classification erronée doit être différentielle (c.-à-d. elle doit mal classifier le devenir de manière préférentielle dans un groupe d'exposition). Puisqu'il est très peu probable que l'utilisation de l'APO elle-même ait pu avoir affecté le diagnostic d'autisme, l'effet proposé du biais de classification erronée sur l'association fallacieuse observée pourrait toujours être valable, si un déterminant de santé lié à l'utilisation de l'APO avait influencé le processus de diagnostic d'autisme et donc, la classification du devenir. Dans une certaine mesure, un tel biais ramène le problème à des hypothèses de facteurs confondants non contrôlés et de causalité.

\section{Qu'est-ce que la causalité?}

Au fil des ans, de nombreux scientifiques ont tenté de circonscrire la causalité à l'intérieur de différents cadres. Sir Austin Bradford Hill a publié des critères bien connus qui ont été utilisés par le Directeur de santé publique (Surgeon General) des États-Unis dans les années 1960 afin d'établir pour la première fois la causalité entre l'exposition au tabac et le cancer du poumon. ${ }^{11}$ Ces critères 
A Un chemin causal biaisé

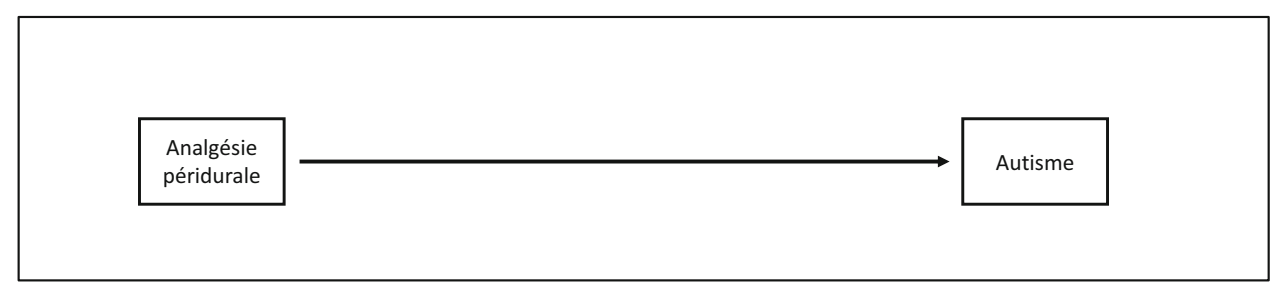

B Un cadre conceptuel causal complexe plus réaliste, mais certainement incomplet

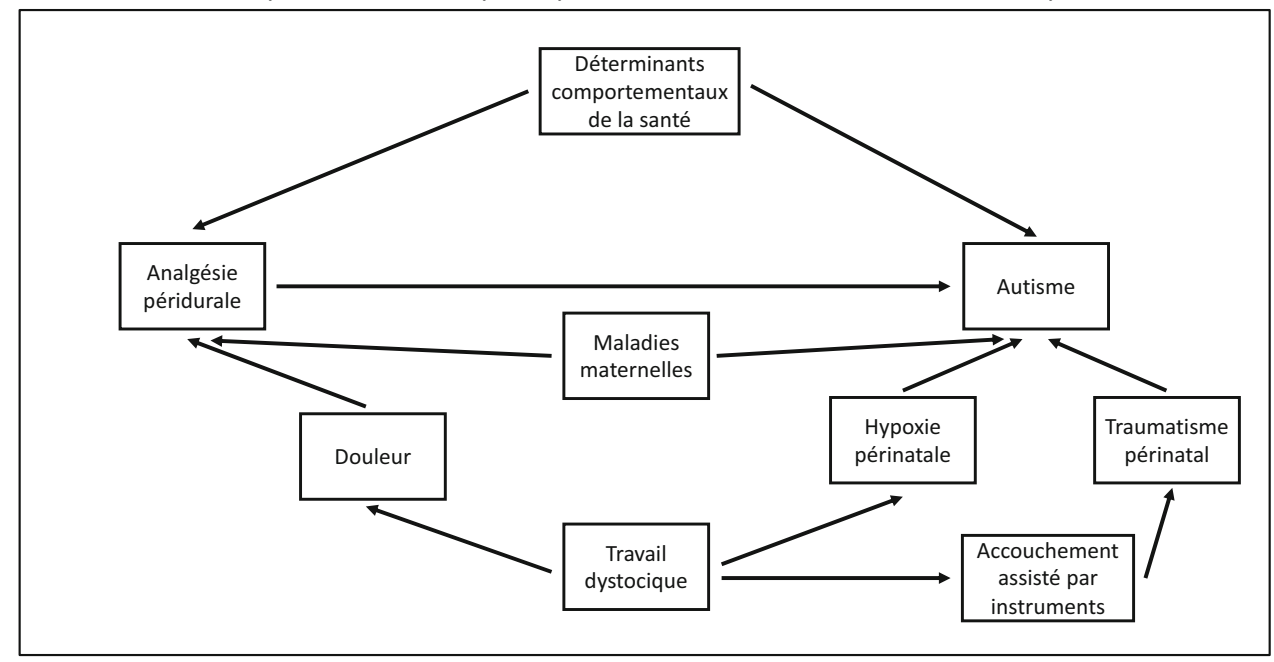

Figure Chemin causal entre l'analgésie péridurale et l'autisme

proposent une évaluation de la causalité par une analyse qualitative des associations observées qui pourrait être considérée comme contournant le problème des facteurs confondants non contrôlés. Certains de ces critères ont été abordés, explorés ou mentionnés dans l'article récemment publié, mais beaucoup d'autres ne l'ont pas été (voir tableau). ${ }^{4}$ Il existe une relation temporelle entre l'APO et l'autisme, puisque l'un se produit avant l'autre. Les auteurs ont trouvé une relation de dose-réponse significative, ce qui constitue un critère causal important. Ils ont exposé la plausibilité biologique de cette association en rapportant des données translationnelles sur la toxicité des anesthésiques locaux et le cerveau en développement. Néanmoins, ces données sont peu nombreuses, proviennent principalement d'études sur les animaux, et utilisent des doses d'anesthésique local beaucoup plus élevées que celles utilisées dans la pratique clinique. En outre, l'association observée est faible, n'a pas été observée ailleurs, n'est étayée par aucune donnée expérimentale et, fait important, comme nous l'avons vu précédemment, est biaisée par d'autres voies causales, y compris leurs analyses exploratoires de dose-réponse dont les facteurs confondants n'ont pas été éliminés, tout comme dans leurs principales analyses. Comme nous l'avons mentionné, des travaux plus récents ont porté sur la causalité sous un angle plus quantitatif, soulignant le fait que les facteurs confondants sont des variables non aléatoires associées à l'exposition et aux devenirs qui pourraient expliquer bon nombre des observations rapportées. ${ }^{6}$ Dans l'inférence causale moderne, de tels facteurs confondants doivent être complètement évalués, indépendamment des critères proposés par Bradford Hill, avant d'établir un lien de causalité.

\section{Impacts sur la santé de corrélations fallacieuses}

Les associations épidémiologiques et écologiques non causales sont courantes. Les vaccins et l'autisme en constituent un exemple bien connu qui a suscité vingt ans de débats et qui a encore un impact sur les interventions de santé publique. ${ }^{12}$ En obstétrique, l'association entre la parité et l'incidence du syndrome de Down chez les enfants a également alimenté le débat. ${ }^{13}$ La réalité non biaisée de cette association n'est pas due à des perturbations physiologiques complexes qui se produisent pendant les grossesses ultérieures, mais s'explique plutôt par l'âge maternel, qui augmente avec la parité. ${ }^{13}$ On croyait autrefois que l'APO était associée à un taux accru d'accouchement par césarienne, mais il est aujourd'hui 
reconnu qu'il s'agit d'une association non causale. ${ }^{14}$ Tout comme dans le cas des associations susmentionnées, de nombreux facteurs étaient en jeu dans cette association. Par exemple, le travail dystocique augmente le risque d'accouchement par césarienne, et est également associé à une douleur accrue pendant le travail obstétrical, augmentant ainsi la probabilité de demander une APO. Il faut donc faire preuve de prudence lors de la réalisation d'analyses à partir de données observationnelles. Il est difficile, mais crucial, de tenir compte des facteurs confondants pour tirer des conclusions significatives fondées sur des études rétrospectives. Dans le cas contraire, de fausses corrélations, que l'on considère comme causales, pourraient mener à des interventions de santé inappropriées, à de l'anxiété chez les patients, voire à des interventions coûteuses qui ne profitent ni aux patients, ni aux fournisseurs de soins de santé.

\section{La communauté anesthésique réagit}

Le jour même de la publication du manuscrit de Qiu et coll., une déclaration conjointe a été publiée par la Society for Obstetric Anesthesia and Perinatology, l'American Society of Anesthesiologists, la Society for Pediatric Anesthesia, l'American College of Obstetricians and Gynecologists et la Society for Maternal-Fetal Medicine afin de rassurer les femmes que l'association entre l'APO et l'autisme n'impliquait aucune causalité. ${ }^{4,15} \mathrm{La}$ même semaine, le Collège royal des anesthésistes et la SCA ont également émis des déclarations pour expliquer aux femmes que cette étude ne prouvait pas un lien de causalité et, surtout, qu'elle ne changeait pas les recommandations actuelles de pratique clinique concernant l'analgésie péridurale pour la douleur du travail obstétrical. ${ }^{5,16}$ Ces déclarations sont le reflet de l'opinion d'experts de premier plan, établie à partir d'une vaste expérience universitaire et clinique, opinion selon laquelle l'association rapportée est imparfaite, non cliniquement significative et potentiellement nuisible pour les femmes.

Il est nécessaire d'analyser et de rapporter les effets de l'APO sur les devenirs à long terme. La physiopathologie de l'autisme n'est pas entièrement comprise et il est également nécessaire d'explorer les associations épidémiologiques afin d'identifier ses causes potentielles. L'exposition à une APO nécessite un processus décisionnel complexe, c'est pourquoi il est difficile de limiter l'analyse de son rôle dans l'autisme. La publication d'associations spécieuses entre l'APO et les devenirs obstétricaux peut avoir une incidence sur les femmes et leur choix de prise en charge de la douleur, ainsi que sur leur volonté d'adhérer à un plan obstétrical complexe qui implique fréquemment l'APO. De tels choix peuvent entrainer des préjudices potentiels et une culpabilité inutile, plus particulièrement dans des sous-groupes vulnérables de la population. Afin d'empêcher la diffusion de telles corrélations fallacieuses, les analyses d'associations ne devraient pas être réalisées en dehors d'un cadre causal clair qui tienne compte de tous les facteurs confondants importants et adéquatement évalués. Dans le cas contraire, il ne serait que trop facile pour les scientifiques de se perdre sur le chemin de la causalité.

Disclosures The authors do not report any conflict of interest. Opinions included in this text are the authors' own. They do not represent the opinion of their affiliated institutions.

Funding statement Dr. Carrier is recipient of a research career award from the Fonds de Recherche du Québec - Santé.

Editorial responsibility This submission was handled by Dr. Hilary P. Grocott, Editor-in-Chief, Canadian Journal of Anesthesia.

Déclaration Les auteurs ne déclarent aucun conflit d'intérêt. Les opinions émises dans ce texte sont celles des auteurs. Elles ne représentent pas l'opinion de leurs institutions affiliées.

Déclaration de financement Dr Carrier est le récipiendaire d'un prix de carrière en recherche du Fonds de recherche du Québec Santé.

Responsabilité éditoriale Cet article a été traité par Dr Hilary P. Grocott, rédacteur en chef, Journal canadien d'anesthésie.

\section{References}

1. Canadian Institute for Health Information (CIHI). Available from URL: https://www.cihi.ca (accessed November 2020).

2. Lim G, Facco FL, Nathan N, Waters JH, Wong CA, Eltzschig HK. A review of the impact of obstetric anesthesia on maternal and neonatal outcomes. Anesthesiology 2018; 129: 192-215.

3. Maenner MJ, Shaw KA, Baio J, et al. Prevalence of autism spectrum disorder among children aged 8 years - autism and developmental disabilities monitoring network, 11 sites, United States, 2016. MMWR Surveill Summ 2020; 69: 1-12.

4. Qiu C, Lin JC, Shi JM, et al. Association between epidural analgesia during labor and risk of autism spectrum disorders in offspring. JAMA Pediatr 2020; . https://doi.org/10.1001/ jamapediatrics.2020.3231.

5. McKeen DM, Zaphiratos V; Canadian Anesthesiologists' Society. Lack of evidence that epidural pain relief during labour causes autism spectrum disorder: a position statement of the Canadian Anesthesiologists' Society. Can J Anesth 2020; https://doi.org/10. 1007/s12630-020-01840-z.

6. Pearl J, Mackensie D. The Book of Why. Basic Books; 2018.

7. Hernan MA, Robins JM. Causal Inference: What If. Boca Raton: Chapman \& Hall/CRC; 2020 .

8. VanderWeele TJ, Ding P. Sensitivity analysis in observational research: introducing the E-value. Ann Intern Med 2017; 167: $268-74$. 
9. Toledo P, Sun J, Grobman WA, Wong CA, Feinglass J, HasnainWynia $R$. Racial and ethnic disparities in neuraxial labor analgesia. Anesth Analg 2012; 114: 172-8.

10. Glezerson BA, Trivedi V, McIsaac DI. On the stated association between labour epidural analgesia and risk of autism spectrum disorders in offspring. Can J Anesth 2021; . https://doi.org/10. 1007/s12630-020-01869-0.

11. U.S. National Library of Medicine. Smoking and Health: Report of the Advisory Committee to the Surgeon General; 1964. Available from URL: https://profiles.nlm.nih.gov/spotlight/nn/ catalog?f\%5Bexhibit_tags $\% 5 \mathrm{D} \% 5 \mathrm{~B} \% 5 \mathrm{D}=$ smoking (accessed November 2020).

12. Wakefield AJ, Murch SH, Anthony A, et al. Ileal-lymphoidnodular hyperplasia, non-specific colitis, and pervasive developmental disorder in children. Lancet 1998; 351: 637-41.

13. Chan A. Invited commentary: Parity and the risk of Down's syndrome - caution in interpretation. Am J Epidemiol 2003; 158: 509-11; discussion 512-3.
14. Segal $S, S u$, Gilbert $P$. The effect of a rapid change in availability of epidural analgesia on the cesarean delivery rate: a meta-analysis. Am J Obstet Gynecol 2000; 183: 974-8.

15. American Society of Anesthesiologists. Labor Epidurals Do Not Cause Autism; Safe for Mothers and Infants, say Anesthesiology, Obstetrics, and Pediatric Medical Societies. Available from URL: https://www.asahq.org/about-asa/newsroom/news-releases/2020/ 10/labor-epidurals-and-autism-joint-statement (accessed November 2020).

16. Royal College of Anaesthetists. No evidence that labour epidurals cause autism. Available from URL: https://rcoa.ac.uk/news/noevidence-labour-epidurals-cause-autism (accessed November 2020).

Publisher's Note Springer Nature remains neutral with regard to jurisdictional claims in published maps and institutional affiliations. 